\title{
Convergencia Digital: Nuevos perfiles profesionales del periodista*
}

\author{
Andrés Barrios Rubio** \\ William Ricardo Zambrano Ayala***
}

Recibido: 2014-09-26 Aprobado por pares: 2014-11 15
Enviado a pares: 2014-10-08 Aceptado: 2014 12-04

\begin{abstract}
Resumen
El periodismo se está transformando debido al desarrollo de las tecnologías de información y de las comunicaciones (TIC) y a la aparición de nuevos cibermedios que están alterando las tradicionales rutinas de trabajo, los contenidos, la producción, distribución y consumo de información. Este nuevo escenario está marcado por la convergencia digital en sus distintas dimensiones y en la multiplicación de nuevas plataformas, herramientas y formatos de recepción, difusión e interacción que invitan a modificar los perfiles profesionales del periodista y a replantear sus nuevas competencias para ejercer la profesión. Para construir el nuevo perfil del periodista, se realizó una investigación cualitativa y cuantitativa a través de encuestas, entrevistas de profundidad y grupos de discusión, aplicadas a los diferentes actores de las empresas mediáticas y a los directores de programas profesionales de periodismo de Colombia, a partir de tres competencias profesionales: empresarial-tecnológica, comunicativa de contenidos y profesional-usuarios. Se concluye con la identificación de competencias del periodista en un contexto digital, entre ellas: producir contenidos, lenguajes y narrativas multimedia; implementar tecnologías y redes; crear formatos informativos e interactivos acordes con las preferencias de la audiencia, y adecuarse al nuevo ecosistema comunicativo.
\end{abstract}

Palabras clave: periodismo, perfiles, interactividad, convergencia, cibermedios.

- Esta investigación es producto de un trabajo en conjunto entre la Fundación Universitaria Panamericana y la Universidad de Bogotá Jorge Tadeo Lozano. El trabajo fue realizado por los grupos de investigación Comunicación y Medios de la Fundación Universitaria Panamericana, y Comunicación-Medicación-Cultura de la Universidad de Bogotá Jorge Tadeo Lozano, ambos clasificados en Colciencias. El artículo es resultado de la investigación: Convergencia digital, nuevos perfiles del comunicador.

** Comunicador social-periodista, especialista en Edición Digital y Multimedia, magíster en Administración de Empresas con especialidad en Dirección de Proyectos, doctorando en Contenidos de Comunicación en la Era Digital. Actualmente, director Emisora Óyeme UJTL, coordinador Programa de Tecnología en Producción Radial y docente asociado II del Programa de Comunicación Social-Periodismo de la Facultad de Ciencias Sociales de la Universidad de Bogotá Jorge Tadeo Lozano. andres.barrios@utadeo.edu.co

*** Comunicador social-periodista, administrador de Empresas, mercadológo y publicista, especialista en Gerencia en Recursos Humanos, especialista en Comunicación Visual, magíster en Comunicación, doctor en Sociedad de la Información y del Conocimiento, posdoctorado en Dispositivos Móviles. Actualmente, director del Programa de Comunicación Social de la Facultad de Comunicación de la Fundación Universitaria Panamericana.williamzambrano@unipanamericana.edu.co 


\title{
Digital Convergence: New Professional Profiles of a Journalist
}

\begin{abstract}
Journalism is changing with the development of Information and Communication Technology (TIC abbreviation in Spanish) and the emergence of new online media that are changing the traditional work routines, content, production, distribution, and consumption of information. This new stage is marked by the digital convergence in its various dimensions and the multiplication of new platforms, tools, and formats reception, dissemination and interaction that invite to change journalists' professional profiles and to give a second thought to their new skills to practice. To build the new profile of the journalist, a qualitative and quantitative research through surveys, depth interviews and discussion groups applied to the various actors of media companies and managers of journalism programs in Colombia were conducted from three professional skills: business-technological, content communicative and users professional. It was concluded with the construction of a map of competencies of a journalist in a digital context, among others, to produce contents, languages and multimedia narratives. to implement technologies and networks, to create informative and interactive formats consistent with the preferences of the audience, and to adapt to the new communication ecosystem.
\end{abstract}

Key words: journalism, profiles, interactivity, interactive, convergence, cybermedia. 


\section{Introducción}

El entorno comunicativo del mundo de hoy en el que las redes sociales y las plataformas de comunicación brindan un nuevo panorama al quehacer periodístico, el profesional del siglo XXI requiere ser capacitado para afrontar la convergencia de medios, así como la migración de las actividades tradicionales al entorno digital. Se habla de un cambio que implica el dominio de nuevos lenguajes, características y comportamientos de los usuarios, así como nuevos esquemas de producción en donde se combinan el texto, el audio y el vídeo bajo una nueva forma de contar historias: la narrativa transmedial.

A decir de Barrios Rubio \& Zambrano Ayala (2014) el mercado se encuentra saturado de artefactos tecnológicos que son portados por unos usuarios ávidos de relaciones y reconocimientos, lo cual se evidencia en las redes sociales y en las comunidades virtuales, y la forma como interactúa dicho público con los medios y los periodistas marca el entorno digital. Es un panorama en el que se redefine el concepto de sociedad, medios de comunicación y el periodismo de tal manera que se replantea el ecosistema comunicativo tradicional, es decir, la audiencia toma protagonismo, está ávida de información pide una fuerte interacción y pasar de mirar de manera exclusiva los componentes locales para aportar experiencias globales.

Los medios tradicionales son conscientes de ello y han ido migrando sus contenidos a la red; algunos los mantienen como un servicio complementario, y otros se han instalado en la plataforma Internet creando una simbiosis mediática, en la cual se generan nuevos modelos de periodismo, unos cibermedios2 en los que surgen otros modelos de comunicación basados en procesos interactivos, otras modalidades informativas, otros modos de narrar los datos, los hechos, las ideas y opiniones, otros recursos expresivos que superan aquella "integración audio-escrito-visual de los medios anteriores hasta constituir un lenguaje multimedia" (Cebrián, 2009:16).

Los cibermedios se conciben como emisores de contenidos que tienen voluntad de mediación entre los hechos y el público, utilizan criterios, técnicas periodísticas, lenguaje multimedia, son interactivos e hipertextuales, se actualizan y se publican en Internet. La finalidad de estos cibermedios es la misma que la de los medios tradicionales: la producción informativa; lo que ha cambiado es la forma en que se lleva a cabo esa producción. Es una etapa de adaptación en la que los medios de comunicación del mundo se están

\footnotetext{
Según Jenkins (2008), transmedia es aquella que atraviesa numerosos tipos de canales mediáticos de forma estructurada e integrada dentro de cada plataforma, haciendo una contribución valiosa y específica a nuestra experiencia total. Es decir, es una nueva forma de narración concebida para contar historias a través de múltiples plataformas y formatos. Aunque históricamente ha habido una tendencia a la redundancia, a la repetición de la misma información en cada canal mediático; la narración transmediática le da la vuelta para que cada plataforma complemente lo que hemos aprendido de los otros.

2 "Ciber" porque usa el lenguaje multimedia, recurre a la interactividad y la hipertextualidad, se actualiza y se publica en la red Internet. "Medio" porque tiene voluntad de mediación entre hechos y público, emite contenidos, emplea criterios y técnicas periodísticas. Campos (2010) concibe los cibermedios como aquellos medios de comunicación social que emplean el ciberespacio como ámbito para la difusión pública de informaciones periodísticas.
} 
reinventando rápidamente en cibermedios, los cuales se convierten en empresas de publicaciones multimedia, crean plataformas, formatos, contenidos y lenguajes; además, logran a través de la convergencia nuevas audiencias y generan diferentes oportunidades para sus anunciantes.

Se hace referencia a una convergencia que a decir de López \& Pereira (2010), citando a Pavlik (2004), es "el acercamiento de todas las formas de comunicación mediatizada en una forma electrónica y digital, impulsadas por los ordenadores" (45). Es decir, las fronteras desaparecen, la gente puede acceder a cualquier contenido en la plataforma digital. Un concepto polisémico que ha sido usado para describir varias tendencias en el periodismo que tienen algo en común: el desdibujamiento de límites entre diferentes medios, habilidades profesionales y roles (Domingo $\&$ otros, 2007).

La convergencia es "un proceso multidimensional facilitado por la implantación generalizada de las tecnologías digitales de telecomunicación" (Salaverría \& García, 2008:35), lo cual afecta al ámbito tecnológico, empresarial, profesional y editorial de los medios de comunicación, propiciando la integración de herramientas, espacios, métodos de trabajo y lenguajes anteriormente disgregados de forma que los periodistas elaboran contenidos que se distribuyen a través de múltiples plataformas, mediante los lenguajes propios de cada una.

Los medios y la sociedad contemporánea están inmersos en una convergencia digital que se puede definir como el flujo de contenidos a través de múltiples plataformas, la cooperación entre diferentes industrias mediáticas y el comportamiento migratorio de las audiencias, dispuestas a ir casi a cualquier parte en busca del tipo deseado de experiencias de entretenimiento (Jenkins, 2008). En este sentido, los mass media apuntan a distribuir sus contenidos en múltiples plataformas, ya que esta estrategia ayuda a incrementar la posibilidad de difusión y consumo de información. Para lograr con éxito la implementación de este servicio es importante pensar también en la integración de las redacciones, con la finalidad de que el medio tradicional y el nuevo compartan la producción y la explotación de los contenidos; de ahí nace el término convergencia periodística.

Convergencia periodística alude a un proceso de integración de modos de comunicación tradicionalmente separados que afecta a empresas, tecnologías, profesionales y audiencias en todas las fases de producción, distribución y consumo de contenidos de cualquier tipo. Según Scolari (2004) esta convergencia incide en múltiples esferas del quehacer periodístico: en las estrategias empresariales, en los cambios tecnológicos, en la elaboración y distribución de contenidos a través de distintas plataformas, en las formas de acceso a los contenidos y en el perfil profesional de los periodistas. Es el inicio del llamado periodismo digital y de la revolución en marcha alrededor de la red de redes y de la reestructuración del sector de la comunicación y de las empresas mediáticas.

El periodismo digital es la especialidad del periodismo que utiliza la convergencia para la elaboración y difusión de contenidos periodísticos, mientras que Quim (2012) lo 
define como el que rompe con la comunicación lineal y unidireccional e implica una serie de cambios fundamentales respecto a las rutinas del periodismo tradicional.

En atención con lo anterior, el objetivo general de esta segunda fase de investigación es identificar y analizar el nuevo perfil profesional del periodista según la convergencia digital de medios. Para tal fin, se aplicarán las tres competencias3 periodísticas presentadas por García (2009) y Salaverría (2010): Competencia empresarial-tecnológica: actuación profesional en un espacio en el convergen industrias de los diversos sectores de la economía que conforman grupos multimedia en el sector de las comunicaciones, y propician la producción de productos comunicativos para la multiplicidad de canales, plataformas y medios. Competencia comunicativa de contenidos: área que pide el dominio de los lenguajes y los referentes semióticos en la producción de productos comunicativos dotados de sentido bajo el ecosistema comunicativo de hoy que propone nuevas rutinas informativas y la producción de material para diversos formatos. Competencia profesionalusuarios: espacio en el que se replantea el quehacer profesional del periodista en el entorno tecnológico, el dominio de herramientas y la interacción con un público que cada día pide mayor protagonismo e incidencia en los productos comunicativos.

\section{Estado de la cuestión}

Se presenta a continuación una revisión bibliográfica de investigaciones concluidas sobre nuevos perfiles profesionales del periodista según la convergencia digital a fin de identificar sus antecedentes, situación actual y prospectiva de su desarrollo y evolución. Se utilizó el método cualitativo por su descripción, argumentación e interpretación, lo que facilitó la recolección de información y datos estadísticos para fundamentar el análisis en función del contexto nacional e internacional, y así clasificar, organizar, describir, codificar y protocolizar las investigaciones realizadas.

Para rastrear sus antecedentes, se acudió a revisiones de libros, artículos científicos, Internet, bases de datos internacionales, universidades europeas, norteamericanas y latinoamericanas. Para ello, se recogió y agrupó coherentemente un buen número de investigaciones nacionales e internacionales realizadas desde 2008 hasta 2013. El número de estudios que trata este tópico es muchísimo mayor, pero se fundamentó en investigaciones y no en disquisiciones teóricas o en artículos descriptivos de experiencias.

Para iniciar el recorrido propuesto se puede hacer referencia a González \& Ortells (2012) quienes desarrollaron una investigación denominada: "Polivalencia periodística

\footnotetext{
3 Se puede definir la competencia como un conjunto multifuncional y transferible de conocimientos, destrezas y actitudes que todos las personas necesitan adquirir en el proceso de aprendizaje para su realización y desarrollo personal, inclusión en la sociedad y acceso al empleo. Deben ser transferibles y, por tanto, aplicables en determinados contextos y situaciones. En esta investigación, se establece que la competencia digital que abarca tanto a las tecnologías de la información como de la comunicación implica el uso de los medios electrónicos para el trabajo y la comunicación. Estas competencias están relacionadas con el pensamiento lógico y crítico, con las habilidades para el manejo de la información y con el desarrollo eficaz de las destrezas comunicativas.
} 
de los profesionales en las redes sociales"; en ella se identificaron, mediante el método cualitativo, las competencias que requiere un periodista polivalente que incorpora el uso de las redes sociales en su labor de informar. Por otro lado, Tuñez, Martínez \& Abejón (2010) realizaron un estudio cualitativo y cuantitativo, titulado: "Nuevos entornos, nuevas demandas, nuevos periodistas", en el cual se planteó una redefinición de sus rutinas productivas, las cuales deben estar orientadas a formar un periodista con especialización tecnológica en multimedia.

Asimismo, Scolari et al., (2008) hicieron una investigación sobre "El periodista polivalente. Transformaciones en el perfil del periodista a partir de la digitalización de los medios audiovisuales catalanes", desde donde se identificaron, mediante entrevistas, las transformaciones en el perfil profesional del periodista a partir de la convergencia digital en las redacciones de radio, televisión, agencias y cibermedios catalanes.

De igual manera, se realizó un estudio sobre la "Aproximación al perfil del periodista en la Posmodernidad" por parte de Arroyo (2011), el cual se centró en cómo el periodista ha tenido que aprender a trabajar con las herramientas de la web 2.0 en el contexto digital que exige hipertextualidad, multimedialidad e interactividad. A pesar de estos cambios, la autora insiste en la necesidad de la formación humanística que es la que le va a dar sustento para comprender la realidad social y, por ende, construir un buen relato. Por su parte, Larrañaga (2008) investigó cualitativa y cuantitativamente acerca de "La práctica profesional en el proceso de convergencia mediática", donde aplicó un estudio de caso al grupo local de comunicación GOIENA Komunikazio Zerbitzuak, Koop, y concluyó que se requiere un periodista polivalente, redactor multitarea, multimedia y dominio de la tecnología digital, gestor de contenidos y organizador de informativos.

En otro estudio, Yezers)ka (2008) se centró en el "Impacto de Internet en el trabajo de los periodistas digitales en Perú", a través de una metodología cualitativa en los principales periódicos nacionales y algunos regionales, y halló como resultados principales que el perfil del periodista digital es el de un profesional joven (menor de 30 años), licenciado en comunicación por una universidad, con conocimientos digitales y con alto sentido humanístico. Desde su perspectiva, López (2012) investigó "La formación de los periodistas para los entornos digitales actuales", y en él se evidenció, el aumento del nivel de formación de los periodistas en las universidades de España, los cuales necesitan habilidades para manejar los entornos de la Web 2.0 o 3.0., saber construir mensajes con los diferentes lenguajes y para diversos soportes, y entender los distintos modelos de organización social y empresarial mediática.

En otra propuesta de estudio, Salaverría \& García (2008) en su investigación "La convergencia tecnológica en los medios de comunicación: retos para el periodismo" analizaron la convergencia en los medios de comunicación y los retos que implica para el periodista pasar de trabajar en un formato con un lenguaje y una rutina, a fusionar todo en uno. Con el auge de las nuevas tecnologías, las empresas informativas se transforman en grupos multimedia en las que integran las salas de redacción y producción, lo cual implica un cambio de mentalidad y, por ende, de rutina de trabajo para el profesional 
de la comunicación. Se presenta fusión de roles: ciertas actividades que en el pasado correspondían a dos o más profesionales, ahora son ejecutadas por uno solo.

Posteriormente, García \& Martínez (2009) identificaron las "Competencias en la formación universitaria de periodistas a través de nuevas tecnologías"; en la práctica realizada con 105 estudiantes de la asignatura de Informática concluyeron que el nuevo profesional de la comunicación necesita trabajar en equipo para lograr un objetivo común para emitir una noticia. Por otro lado, Castellón \& Jaramillo (2009) investigaron las "Competencias digitales para periodistas", los cuales requieren habilidades digitales interactivas con la audiencia, inteligencia colectiva capacidad para saber navegar transmedia y liderar trabajo en equipo en red.

El Centro Knight para Periodismo en las Américas (2009) resume una serie de demandas planteadas por la Fundación Nuevo Periodismo Iberoamericano (FNPI) sobre la formación que debieran tener los nuevos periodistas. Lo anterior contrasta con Arroyabe E Blanco (2005) en su investigación "Cómo perciben los periodistas su profesión", donde resaltan que los estudiantes de cCmunicación no están tan preparados en América Latina para enfrentar las realidades de la profesión en la región, según las percepciones que tienen los profesionales de los medios de cuatro países del continente: Uruguay, El Salvador, República Dominicana y Argentina.

Para finalizar este rápido recorrido internacional se debe hacer referencia a Deuze (2005) y su investigación titulada "What is journalism?: professional identity and ideology of journalists reconsidered", donde analiza la identidad profesional de los periodistas. Concluye que este profesional tiene pretensiones de exclusividad en la sociedad basado en su ideología ocupacional, pretensiones que debe cambiar en el contexto de los acontecimientos culturales y tecnológicos actuales. Desde su perspectiva, Morales (2005) en "Tendencias y debates actuales en el periodismo digital", plantea los cambios que ha tenido el oficio en las dinámicas de la producción de información, las rutinas del periodista, sus ámbitos laborales y legales, y en los procesos empresariales que lo circundan.

En Colombia, actualmente se presentan algunas propuestas de estudio sobre convergencia digital, que empiezan a ser el cimiento de futuras investigaciones, abriendo un campo poco desarrollado en el país; sin embargo, queda todavía un largo camino por recorrer y asentar esta disciplina. En este contexto, pocas son las investigaciones, congresos y eventos que se han realizado sobre el nuevo perfil profesional del periodista según la convergencia digital.

Las investigaciones referenciadas nos ubican en el caso particular del perfil del periodista según la convergencia; se encontró una variedad de análisis que destacan la "revolución" de la profesión del periodismo a partir de la digitalización de los soportes hasta perspectivas que apuntan críticamente a dichos enfoques. Los estudios resaltan los nuevos conocimientos que deben adquirir los profesionales en la convergencia digital, pero no concretan ni marcan los perfiles según las competencias propuestas en la presente investigación. En consecuencia, se formulan las siguientes preguntas: ¿Qué 
transformaciones están experimentando los medios tradicionales según la nueva tecnología convergente? ¿Qué nuevos contenidos, narrativa, lenguajes y estilos requieren estos medios digitales? ¿Cuáles son las nuevas competencias profesionales del periodista que demandan los cibermedios?

La puesta en marcha para responder al objetivo, las tres competencias y los interrogantes planteados significará una oportunidad para las empresas de comunicación tradicionales que están en su proceso de transformación digital y para aquellos medios que están en la red, los cuales requieren de un nuevo perfil profesional del periodista. De igual forma, los resultados de esta investigación serán insumos para los Programas de Periodismo que están modificando sus planes de estudio. Por tales razones, el tema interesa porque en el país no existe una investigación equivalente, salvo pequeños estudios que abordan, de manera general, el periodismo digital, pero que no han tenido en cuenta los cambios que están experimentando los medios en general y el periodismo en particular.

Esta investigación se fundamentó en una buena conducta de indagación y trabajo de campo, respaldada por la ética, la calidad, la responsabilidad y la independencia de los autores, los cuales no tuvimos conflictos de intereses intelectuales ni de financiación durante todo el diseño y desarrollo del presente trabajo, y siempre nos basamos en una cultura de respeto, conocimiento y difusión autorizada del conocimiento.

\section{METODOLOGÍA}

Se realizaron 120 encuestas de una muestra de 537 periodistas, los cuales fueron seleccionados con los siguientes criterios: llevar mínimo cinco años trabajando en los medios de comunicación que han sufrido la convergencia digital y que estuvieran activos en el período de la realización de la investigación (10 de noviembre de 2012 a mayo 30 de 2013), en prensa, radio y televisión en Bogotá, Cali, Medellín y Barranquilla, ciudades donde se concentra la mayoría de estos medios4 (Rey, 2013) y que estuvieran en rango de edad entre los 21 y los 60 años, con características de nativos5 y migrantes6 digitales. En cuanto a los decanos y/o directores de Programas de Periodismo se escogieron aquellos que estuvieran activos en las ciudades mencionadas. Para el cálculo del tamaño de la muestra, al igual que en la primera fase de estudio, se aplicó la fórmula de Mcdaniel $\varepsilon$ Gates (2009); Hair et al., (2011):

Donde $N=537, Z=1.96$ para un grado de confiabilidad del $(95 \%), p=$ Probabilidad de éxito $(90 \%), q=$ Probabilidad de fracaso $(10 \%)$, e $=\%$ de error muestral de $(8 \%)$,

\footnotetext{
$4 \quad$ Aquellos cuyo único contenido web corresponde a la misma información producida para sus plataformas originales (radio, prensa o televisión). Recurren a mecanismo como la publicación de sus versiones en diferentes formatos o la posibilidad de acceder a contenidos en vivo o en diferido a través de Internet.

5 También llamados Homo Sapiens Digital, hace referencia a todas las personas nacidas durante la época de los 80 y 90, periodo en el cual ya existía la tecnología digital. Son las nuevas generaciones habituadas al uso de los medios de comunicación como lo son la telefonía celular, el internet, la prensa, la televisión y la radio.

6 Son personas entre los 35 y 50 años, que han aprendido a usar las tecnologías y adecuarse a los nuevos sistemas de convergencia digital, entre otros a los ordenadores, los celulares, Internet y redes sociales.
} 
$\mathrm{n}=$ muestral. Una vez aplicada la fórmula se obtuvo un N muestral de 120 encuestados (tabla 1).

Tabla 1. Distribución de la muestra

\begin{tabular}{|l|c|c|c|c|c|c|c|}
\hline Cargo Medio & $\begin{array}{c}\text { Jefes de } \\
\text { redacción }\end{array}$ & $\begin{array}{c}\text { Editores } \\
\text { generales }\end{array}$ & $\begin{array}{c}\text { Periodistas } \\
\text { y comunica- } \\
\text { dores }\end{array}$ & $\begin{array}{c}\text { Estudiantes } \\
\text { y egresados }\end{array}$ & $\begin{array}{c}\text { Docentes de } \\
\text { comunicación }\end{array}$ & $\begin{array}{c}\text { Decanos- } \\
\text { directores }\end{array}$ & Totales \\
\hline Prensa & 5 & 6 & 4 & 5 & 4 & 7 & 31 \\
\hline Radio & 6 & 5 & 5 & 5 & 5 & 6 & 32 \\
\hline Televisión & 5 & 5 & 5 & 6 & 5 & 9 & 35 \\
\hline Internet & 4 & 3 & 3 & 4 & 4 & 4 & 22 \\
\hline Totales & 20 & 19 & 17 & 20 & 18 & 26 & 120 \\
\hline
\end{tabular}

Fuente: elaboración propia.

Las encuestas fueron revisadas y validadas antes de ser enviadas vía Internet. Las respuestas de las encuestas y de las entrevistas fueron analizadas calculando las distribuciones de las preguntas cerradas y abiertas de una manera inductiva, con el fin de encontrar temas comunes y opiniones generalizadas. Asimismo, se empleó un enfoque inductivo y deductivo mediante la técnica de grupos de discusión y análisis, integrados en 18 grupos, cada uno de cinco periodistas, para un total de diez sesiones, cada una de una hora en promedio, registradas en grabadoras y en libreta de apuntes.

Dichos instrumentos fueron diseñados y validados según la metodología propuesta por Salazar y Sepúlveda (2011), la cual se aplicó en tres fases: diseño y realización del estudio, resultados y discusión, y conclusiones. Se elaboraron los estándares para puntuar y procesar la información en Excel a través de tablas dinámicas que permitieron su tabulación. Los datos se cotejaron con los rangos de cada ítem, con el propósito de sistematizar y analizar cada una de las variables.

Se presentaron algunos obstáculos en la consecución de dos encuestados, y dos entrevistados que no cumplieron con la totalidad del tiempo requerido para profundizar el tema propuesto, lo que condujo a ampliar la diversidad de fuentes primarias mediante testimonios orales, documentación escrita y registros audiovisuales, los cuales fueron importantes referentes para la construcción del objeto de estudio debido a que hubo estrecha correspondencia entre los datos de ubicación y la caracterización de las variables de la información obtenida; su organización y evaluación se constituyó en soporte argumental para presentar los resultados con parámetros e indicadores.

\section{RESULTADOS Y DISCUSIÓN}

Competencia empresarial-tecnológica: el 89 \% afirmó que las empresas mediáticas se están convirtiendo en un solo grupo multimedia, haciendo una migración íntegra de 
canales y soportes de distribución de información en uno solo. El 95 \% respondió que estas organizaciones tratan de integrarse como cibermedios para buscar nuevas audiencias con el fin de alcanzar una mayor rentabilidad. El 98 \% dijo que las empresas mediáticas tradicionales están integrando los medios y las redes para identificar nuevos mercados y nuevas modalidades de control sobre el ecosistema comunicacional. El 78 \% manifestó el interés de estas empresas digitales por hacer presencia a través de los dispositivos móviles.

El 99\% expresó que las empresas sí están replanteando sus medios tradicionales de acuerdo con la nueva tecnología convergente, el 95 \% aseguró que debido a esta transformación se están realizando nuevos procesos de comunicación e interactividad, el 94 \% sostiene que estos medios convergentes generan nuevos modelos de negocios, producen rupturas tecnológicas, crean nuevos hábitos de consumo e imponen otras formas de relacionarse. El 94 \% evidenció un crecimiento del consumo de medios digitales mientras decae el de los tradicionales. El 98 \% concluyó que los medios convergentes experimentan otros espacios, tiempos, de expresión y de consumos.

Estas empresas mediáticas tradicionales en su transformación cuentan con menos gente, recursos y tiempo para investigar; se están instalando en los sitios Web, donde se constituyen nuevas formas de relación social e institucionales, nuevas categorías de aprehensión informativa, social y cultural mediante narrativas breves, registros cortos y datos sustanciosos en contenido y estilo profundo y bien contado, dirigidos a una audiencia interactiva y a unos anunciantes y patrocinadores que esperan que dicha producción sea de alta aceptación por parte del usuario.

La tendencia, según los grupos de discusión, muestra que esta competencia empresarial-tecnológica integrada en los cibermedios tiene protagonismo mientras se reducen los medios análogos; es decir, estos últimos no están desapareciendo, solamente se están adaptando a las nuevas realidades sociales, a los cambios estructurales del sistema mediático y a los procedimientos, los formatos, los lenguajes y las narrativas. «Entramos en la revolución digital que es un cambio profundo de las empresas no solo para las industrias mediáticas, la comunicación y el periodismo, sino también para la humanidad», aseguró Roberto Pombo, director del diario El Tiempo.

Según Anderson (2012) la competencia empresarial-tecnológica reflejada en la convergencia multimedial exige: más velocidad y dinamismo de las informaciones, necesidades de autocomprensión y de interacción; un discurso emergente reflejado a través de producciones innovadoras de mensajes y de interpretaciones; es decir, como expresa Henry Delgado editor del diario El País-Cali. com.co, "es un espacio de diálogo, de interacción y de imaginación; otras formas de producción de información socialmente relevante que atienda no solo a los mercados en general, sino a las necesidades y demandas de sectores sociales específicos"; una integración de diversas informaciones y lenguajes para responder a esta nueva tecnología digital. La sobreabundancia de información no garantiza una sociedad comunicada: hay que saber seleccionar los contenidos precisos para cada situación, medio y audiencia, considera Minsky (2010). 
Por otro lado, el 97 \% afirmó que estos cibermedios introducen otras formas de comunicación basadas en los procesos de interactividad para facilitar el diálogo activo entre audiencia. A la pregunta si la competencia empresarial-tecnológica de los medios tiene ventajas, el 97 \% respondió que sí, por su actualización, accesibilidad, hipertextualidad e hipermedialidad.

Juan Gómez, director de El Colombiano.com.co, sostiene que "estas transformaciones técnico-comunicativas propician la incorporación de los medios tradicionales a medios digitales y a la creación de otros dentro de la red", lo cual ocasiona cambios estructurales en el sistema mediático general, ampliando el abanico a otros medios digitales hasta llegar a una hiperespecialización tanto en los contenidos y la información como en el entretenimiento y formas de persuasión.

Lo anterior es la muestra de una revolución tecnológica que ha cambiado a las personas y su forma de informarse. «La gente ya no solo es consumidora sino productora de contenido, pero eso no va a desplazar al periodista, expresa María Sánchez, comunicadora de la-republica.com. Fidel Cano Correa, director del diario El Espectador, afirma que no se trata de competencias nuevas, las del periodismo digital, sino de las destrezas de siempre bajo las exigencias que han planteado las tecnologías de la información.

Competencia comunicativa de contenidos: el 87 \% asevera que el enfoque y la forma como se desarrolla un contenido para determinada audiencia han cambiado, principalmente en lo creativo y su comunicación efectiva. El 84 \% evidencia que estos medios no utilizan fichas de registro de contenido. El 78 \% respondió que los formatos como entretenimiento, informativos, culturales y deportivos son de mayor frecuencia, más concretos, pero con menor calidad y originalidad. El 96 \% expresa que el estilo o manera en que un periodista presenta un contenido ha cambiado, ahora se diseñan a la medida del usuario, primero está la información y segundo la interacción.

El $98 \%$ afirma que los contenidos son los mismos de los medios tradicionales, pero "reciclados" en un solo. El 95 \% identifica lo mencionado como una convergencia periodística, la cual incorpora otras modalidades de cubrimiento informativo, de estilo de presentación y de consumo, lo cual confluye a una sola redacción para producir contenidos en múltiples plataformas.

Chelo De Castro, periodista de El Heraldo.co, dice que los cibermedios abren los contenidos a otros campos en los que se junta lo relevante con lo cotidiano, lo de interés general con el particular. Carlos Cadena Gaitán periodista de El Mundo.com, sostiene que los nuevos medios incorporan otros contenidos relacionados directa o indirectamente con la información; suelen agruparlos por secciones, en parte, coincidentes con las de los medios tradicionales, y en parte, diferentes por la tendencia a otorgar mayor papel a campos informativos especializados, inclusive de entretenimiento, juegos y compraventas. Se rompe con la división por secciones; cada información adquiere un sentido particular. 
El 87 \% dice que en estos cibermedios el lenguaje es más culto, respetuoso y dinámico; sus narrativas no son disgregadas y rígidas, se articulan y se combinan a través de hipervínculos. El 97 \% considera que estos medios presentan tres géneros periodísticos con mayor interés: la noticia, la entrevista y la opinión, mientras que los de menor énfasis son crónica, necrología, reportajes y perfil. El 96 \% dice que hay cambios en la concepción de la información y en los modelos narrativos. El 82 \% considera que estos medios digitales emplean nuevos modelos de comunicación interactiva y emergente; su producción, programación e intercambios de contenidos y de lenguaje son más exigentes porque se necesita profundizar en la participación en diferentes plataformas y dispositivos digitales.

Los grupos de discusión expresaron que los medios tradicionales paulatinamente están entrando en la convergencia mediática; según Ricardo Ávila director de Portafolio. Co, ahora la tendencia más innovadora es la de trasladar el universo de los medios digitales de las redes fijas a las redes de comunicación en movilidad propiciadas por la telefonía móvil, lo cual experimentan otros espacios y tiempos, otras formas expresivas, otros consumos y, en suma, otras formas de comunicación, de narración, interactividad7 e hipertextualidad8.

Rafael Ortega de la revista Dinero.com, afirma que debido a esta convergencia me diática, se modifica el sistema de información y la consecución de datos, lo cual genera un tratamiento técnico-expresivo de los datos e informaciones compartidas por todos los nuevos sistemas cibermediáticos. Se ha multiplicado el número de fuentes, se requiere más material multimedia, periodistas digitales, competimos con los propios lectores en labores como el análisis, la opinión o la propia información.

Competencia profesional-usuarios: el 85 \% afirmó que estos medios digitales son espacios en permanente construcción a través de la interacción, cuyo objetivo es buscar una identidad de sus usuarios, la cual está en etapa inicial. El 92 \% dijo que hay una relación estrecha y dinámica con las audiencias, es más amplia y menos diversificada. El 81 \% reconoció que los recursos más usados son los foros, grupos, blogs, chats, llamadas y el correo electrónico. El 87 \% utilizó las redes sociales como Facebook y Twitter; con menor porcentaje, Flickr, Skype y YouTube. El 98 \% expresó que con la convergencia de estos medios se pasó al estudio de las comunidades virtuales, sus comportamientos y la producción informativa.

El 87 \% aseveró que el desarrollo de nuevas relaciones entre los usuarios con apoyo de redes sociales y de herramientas Web 2.0 y 3.0 ha sido importante para vincular más al usuario con el medio. El 99 \% manifestó que los cibermedios generan procesos interactivos a través de nuevas formas de expresión, de narrativas y de sinergias entre los diversos medios y sus audiencias. El 95 \% consideró que los consumidores de estos medios di-

Según Rost (2006) la interactividad es "la capacidad gradual que tiene un medio de comunicación para darle a los usuarios un mayor poder tanto en la selección de contenidos (interactividad selectiva) como en las posibilidades de expresión y comunicación (interactividad comunicativa)" (195).

8 Salaverría (2005) define la hipertextualidad como un documento polimorfo que se construye enlazando distintas piezas textuales y/o audiovisuales, interconectadas entre sí gracias a la tecnología digital. 
gitales no se conforman únicamente con leer textos, sino que buscan complementar su información con vídeos, audios, animaciones e infografías. El 14 \% de los periodistas dijo que se intenta hacer un "cibermedio" a la carta": la audiencia escoge los contenidos de su preferencia viendo el menú e insinuando su propia programación.

Según Julio Sánchez Cristo, director de la W Radio, ahora son las audiencias o lectores los que toman un protagonismo clave porque son los que cuentan la versión de sus hechos, cuestionan e invitan a los periodistas a difundirlos. Ahora, gran parte del poder en los medios se mueve desde el individuo, que ya no es solo un consumidor de información, sino que es un productor, expresó Vicky Dávila, directora de noticias de la F. M. Radio.

Las audiencias de estos cibermedios tienen características, comportamientos, demandas y formas particulares distintas a las de los medios tradicionales; su fin es presentar la información según audiencias determinadas. Fernando Molina, presidente de RCN radio, opina que los periodistas digitales como mínimo deben dominar las aplicaciones en línea denominadas software social a fin de interactuar con sus audiencias según sus preferencias.

Rafael Poveda, exdirector de Cable Noticas, dice sobre los usuarios: "los nuevos medios de comunicación determinan una audiencia segmentada y diferenciada que, aunque masiva en cuanto a su número, ya no es de masas en cuanto la simultaneidad y uniformidad del mensaje que recibe". Ricardo Alarcón, presidente de Caracol Radio expresa que "debido a la multiplicidad de mensajes y fuentes, el usuario se ha vuelto más selectivo".

Dicha relación entre audiencia y periodistas es la interactividad más importante en un cibermedio. Comentar, opinar y calificar un contenido es más significativo que solo consumir un texto; como afirma Cortés (2003), la consecuencia es "la proliferación de audiencias fracturadas en unidades más pequeñas: más nichos, y menos masas" (67), donde el periodista deja atrás el concepto de emisor activo y se convierte en un mediador9 de espacios más democráticos y participativos. Estos nuevos usuarios, a pesar de encontrarse caóticamente distribuidos en una red descentralizada, han venido formándose en comunidades virtuales.

El 45 \% afirmó que en estos cibermedios se realizan consejos de redacción; el 88 \% expresó que se debe trabajar para tres o cuatro medios a la vez porque estos convergen en uno solo; los horarios son más prolongados y la versatilidad periodística es más exigente. El 89 \% dijo que la creatividad es indispensable porque hay que saber presentar con similares contenidos trabajados desde los mismos géneros para diferentes audiencias. El 22 \% estableció un diálogo con sus compañeros periodistas para ampliar de manera significativa métodos de trabajo con mayor participación de la ciudadanía.

El 88 \% consideró que las labores periodísticas han cambiado en la arquitectura de la información, diseño, organización del medio y en su finalidad de trasmisión en diferentes plataformas a través de la Web. El 98 \% aseveró que se han acelerado los procesos de

Mediador es hacer posible que unos hombres reconozcan a otros, y ello en doble sentido": que les reconozcan el derecho a vivir y pensar diferente, y que se reconozcan como hombres en esa diferencia. 
publicación según el modelo de redacción digital del medio que opera 24 horas continuas los siete días de la semana; el 79 \% afirmó que se ampliaron las franjas horarias mañana, tarde, noche y madrugada para cubrir la información y actualizarla permanentemente, lo cual exige mayor gestión en la interactividad y en la edición multimedia.

El $88 \%$ de los periodistas entrevistados aseguró que los cibermedios concentran más tareas en el proceso de producción en un solo periodista, mientras que anteriormente en los medios tradicionales las realizaban distintas personas. El 94 \% coincidió que las rutinas de producción de información no se diferencian en gran parte, pero sí se aumentan las horas de trabajo (6 a.m. a 2:00 p. m., de 2:00 a. m. a 9:00 p. m., de 9:00 p. m. a 6:00 a. m.).

Según los grupos de discusión los cibermedios modifican las rutinas de producción de información, y se organizan de acuerdo con las necesidades de cada medio de comunicación. "El periodista personaliza sus notas, las cuales no pasan por filtro de editores para las correcciones, porque deben publicarse lo más pronto posible", afirmó Hernán Peláez, director de la Luciérnaga de Caracol Radio. Es decir, las rutinas determinan lo que el medio es y lo que podría llegar a ser, responden a la inmediatez, a la omnipresencia, al acceso global a la información y a nuevas características multimedia. Armando Neira, director de la Revista Semana.com, complementa "que se suman los sistemas multiplataforma de gestión de contenidos y se cambia el monólogo por diálogo; esto orientado a informar, formar y motivar".

\section{NUEVOS PERFILES PROFESIONALES DEL PERIODISTA}

Competencia empresarial-tecnológica: se requiere un periodista con aptitudes y actitudes para adaptarse a los cambios de las empresas mediáticas en un solo grupo multimedia; que conozca las características de la estructura de medios digitales; aplique elementos innovadores que estén en coherencia con las necesidades de las nuevas audiencias; desarrolle competencias para ejercer la interactividad en cualquier medio, y propicie la reflexión y la crítica sobre los nuevos paradigmas de la dimensión empresarial y del ecosistema de la comunicación. Hoy estas empresas exigen un periodismo integrado, es decir, una polivalencia funcional, mediática y temátical0, un experto capaz de producir contenidos en diferentes lenguajes en medios, soportes y aplicaciones.

Un periodista multimedia, capaz de tratar la información en multipantallas; genere nuevas propuestas y paradigmas en congruencia con las necesidades del sector comunicacional; domine la multimedia; seleccione mejor la información según el interés de la audiencia; use eficientemente la plataforma tecnológica y los dispositivos móviles de acuerdo con los hábitos de consumo de los usuarios; asuma sus roles de multiárea y multiplataformall; conozca la arquitectura de la información del sitio donde trabaja, y se

10 Para Salaverría y Negredo (2008), la polivalencia funcional, la empresa reclama un periodista que asuma labores distintas de su especialidad; la mediática, el periodista informa sobre un suceso a través no solo de un medio sino de varios; y la temática, trabaja como periodista en varios medios al mismo tiempo.

1 Según Campos (2010) el periodista multiárea asume múltiples labores de redacción, fotografía, edición, etc., que antes eran realizadas por distintos profesionales. Este periodista elabora y difunde sus informa- 
familiarice con el entorno Internet, la búsqueda de información, la variedad de fuentes y la interactividad.

Se atraviesa por un panorama que exige un periodista con perfiles polivalentes; manejo de la interactividad y la hipertextualidad debido a nuevos prosumidores (productoresconsumidores); que domine paquetes de ofimática, navegación y búsquedas avanzadas; utilice la red como fuente de información y de noticias; conocimiento de los medios de comunicación y tecnologías multimedia orientadas al desarrollo de contenido; conozca portales, blogs; use programas de edición; utilice el periodismo transmedia; y cree estrategias de circulación de información y desarrollo de interactividad en soporte digital.

Un periodista competente en el uso de equipos digitales de registro visual, realización y edición (cámara de fotografía y de vídeo) y sonoro (micrófonos y grabadoras), prensa (hipertextualidad) e Internet (interfaces); capacidad de compresión de vídeo y agilidad para su transporte y descarga; dominio de modalidades de navegación, enlaces y plataformas; manejo de blogs y de redes sociales; experto en SEO (Optimización para Motores de Búsqueda)12; diestro en leer pantallas, formatos virtuales, tablas de datos y escenarios gráficos diseñados para la captura y contextualización de la información; atento a la actualización constante de las noticias a través de sistemas RSS; y capaz de hacer una lectura multimodal y con multiplicidad de códigos.

Competencia comunicativa de contenidos: exige un periodista multiplataforma o polivalente que multiplique sus tareas a lo largo del proceso de producción, edición y publicación de la información en distintos formatos; conozca el Sistema de Gestión de Contenidos (CMS) y, sobre todo, la organización de la redacción; reformule las diferentes rutinas periodísticas; sepa circular la información en diferentes canales, contar historias breves y aplicar el periodismo de datos; maneje nuevos formatos de mínima duración producidos para la red; que pase de la narración lineal escrita o audiovisual a otra multimedia e interactiva; emplee niveles de apropiación crítica y creativa de los lenguajes; interrelacione contenidos y tratamientos con otros; colabore para que el usuario seleccione el contenido; integre todos los medios a la navegación e hipertextualidad, y amplíe la información a través de hipervínculos.

El campo profesional de hoy requiere de un periodista con conocimiento de los distintos tipos de formatos y géneros para medios digitales; interpretativo; analítico, reflexivo y crítico; que desarrolle los lenguaje propios para cada plataforma; articule el uso escrito y audiovisual con su función comunicativa en interacción con los demás elementos expresivos; que interprete las categorías estéticas como innovación formal y temática, originalidad, estilo, escuelas o tendencias de los nuevos medios de comunicación; redacte mensajes de calidad con el fin de llegar a los blogs y sitios de noticias.

ciones a través de múltiples canales; para ello, ajusta sus textos a las características de cada medio.

12 La tarea de ajustar la información de las páginas que se pretenden hacer aparecer en primeras posiciones de los resultados es conocida como SEO, sigla en inglés de Search Engine Optimization, o sea, 'Optimización para motores de búsqueda'. 
Se necesita un periodista que maneje géneros, lenguajes y narrativas, distinga un mensaje escrito-audiovisual en función de la categoría y el género al que pertenece; produzca información en tiempo real; capaz de reaccionar de manera rápida en la cobertura informativa de eventos de última hora; relacione imágenes de manera creativa; identifique las características expresivas específicas de cada medio de comunicación; domine la escritura, la imagen y/o el sonido en cualquier clase de soporte y de canal; capacidad de lectura interpretativa, argumentativa y crítica de los mensajes; analice, comprenda, asocie y aplique los diversos textos escritos, verbales y visuales en forma sencilla y articulada para diferentes medios, soportes y plataformas digitales.

Competencia profesional-usuarios: el periodista deberá propiciar una interacción continua con los usuarios; ser hábil al investigar, seleccionar y publicar información, conocer de estudios de audiencia; anticiparse al interés del usuario; que escuche y seleccione los paquetes de contenidos sonoros, textuales y visuales propios de la convergencia multimedia; valore los elementos emotivos, racionales y contextuales que intervienen en la recepción; capaz de enfrentarse a una audiencia global activa que no solo lee sino que comenta las noticias y da puntos de vista. De igual forma, el periodista debe conocer a profundidad el social media, dar cuenta de quién está visitando su medio, cómo llegar allí y quienes están más en la red; su fin es ganar públicos y crear espacios de mayor acercamiento con sus audiencias.

Se necesita un periodista que genere innovación a través de la investigación; abierto a una renovación continua de conocimientos periodísticos, con sólida formación permanente; apto para enfrentarse a la superabundancia de información y con suficiente agilidad para seleccionarla; capaz de generar mensajes informativos adaptados a las características de la red y según las rutinas de producción de los medios digitales; comprender los ritmos, las lógicas, los flujos y las redes de los cibermedios; adaptar los cambios de los lenguajes; articular la aparición de nuevos dispositivos para el transporte y procesamiento de información, y consciente de que ya no es tan solo un productor de contenido sino que pasa a ser un arquitecto de la información interactiva.

Un periodista con cultura general, creatividad, pensamiento crítico y analítico; que se centre en asimilar la transformación de los medios; de adecuar las velocidades de transmisión, acortar la ruptura de las distancias; competente en la convergencia de medios para afrontar las necesidades y cambios que configuran el ecosistema comunicativo; que propenda por una sociedad interconectada a través de la participación activa; competitivo, autónomo y autogestor; capaz de trabajar colaborativamente; crítico, analítico, interpretativo, propositivo y argumentativo; que promueva el desarrollo humano, social e individual; fomente los valores con espíritu emprendedor que lo motive a alcanzar la excelencia; asuma con rigor, imaginación, conversación, tecnología, experimentación y humildad nuestra profesión, y comprometido con el mejoramiento de la calidad de vida de todos los miembros de su comunidad y del desarrollo regional y del país. 


\section{CONCLUSIONES}

Las transformaciones de los modelos de negocio de las empresas mediáticas tradicionales no se dieron porque apareció Internet, sino porque la sociedad se está transformando. Es justamente porque las sociedades están cambiando que las tecnologías aparecen y se modifican. No se transforma solamente el soporte tecnológico del periodismo, sino también los medios de comunicación y los modelos de negocio que intervienen en los mismos.

Las transformaciones que están experimentando estos medios tradicionales, según la nueva tecnología convergente, se evidencian en las nuevas rutinas de producción de información, en las formas de trasmitir la noticia, la organización del trabajo en las salas de redacción, los roles, los alcances y la correlación con la adaptación a los cibermedios y sus lógicas internas. La mayoría de redacciones en los medios están desapareciendo porque el periodista trabaja desde la casa, y envía la información por modem. Sus únicos interlocutores son una pantalla, el medio y el oficio de periodista, los cuales se están convirtiendo en un sector terciario de prestación de servicios que debe competir en un el mercado con visión de negocio, entretenimiento e información.

Por tales razones las carreras de Periodismo deberían pensar que las sociedades y las empresas mediáticas necesitan profesionales que entiendan que no es suficiente con cambiar las formas de narrar y empaquetar los contenidos y la información en nuevos cibermedios y dispositivos digitales, sino que debe cambiarse la forma de pensar, de conocer, de avanzar hacia una epistemología del periodismo que dé cuenta de las identidades sociales, de las subjetividades y de los modos de incorporarse a los procesos de socialización, y a la definición misma de la vida cotidiana y sus permanentes transformaciones que experimenta el periodismo gracias a la convergencia digital. Como señala García (2009), la formación de los periodistas tiene un importante componente de saberes humanísticos, que han de estudiarse teniendo en cuenta el fin específico del periodismo, pero también una enseñanza práctica reflexiva y artística.

Lo anterior exige que el nuevo periodista colombiano, según la convergencia digital, tenga las siguientes competencias:

- Ser un profesional polivalente, multimedia y ciberperiodista;

- Con conocimiento de las tecnologías y los dispositivos móviles, dominio de la fotografía, vídeo, sonido y edición de información en la red;

- Con interacción a través de las redes sociales y actualización ágil de la información para diferentes plataformas;

- Capaz de producir información dinámica y bien documentada a la misma velocidad de los consumidores, con calidad, veracidad, ética y relevancia de ser publicada;

- Redactor multitarea y multimedia, capaz de difundir una información en diferentes formatos y géneros periodísticos; 
- Con conocimientos de cultura general;

- Trabajo interdisciplinario en red (reportaría on-line);

- Habilidad para comprender e interactuar con otras culturas;

- Formación de pensamiento crítico y analítico;

- Destrezas para recopilar información, elaborar guiones, editar contenidos, actualizar el sitio web;

- Ético, responsable socialmente, excelente persona y extraordinario profesional.

Estas competencias exigen abordar la profesión del periodista desde varios frentes: la articulación entre los aspectos disciplinares, profesionales y la formación integral, no únicamente digital, sino alguien capaz de concebir el proceso entero, dar cuenta de lo que se quiere comunicar, de los públicos a quienes se dirige y de los discursos interactivos en que deba expresarse. Estas razones hacen pensar en investigar los cibermedios desde la concepción, escritura, producción, realización y dirección de proyectos con alto contenido social. Por tales motivos, se pregunta: ¿Qué contenidos se deben crear para nuevos medios digitales, para satisfacer las necesidades y las expectativas de las empresas mediáticas? ¿Qué lenguajes, formatos y géneros narrativos al servicio de las comunidades, la audiencia y los públicos con sentido social y de convergencia digital se necesitan? ¿Qué nuevas morfologías y dinámicas de la comunicación en la red, se están desarrollando en medio de las tensiones de la multimedialidad, la convergencia y la interactividad?

\section{BIBLIOGRAFÍA}

Anderson, Ch. (2012). The Long Tail: Why the Future of Business Is Selling Less of More. New York: Hyperion.

Arroyabe, J. E Blanco, I. (2005). Cómo perciben los periodistas su profesión: entre el agotamiento y la fascinación. En Investigación y desarrollo. Vol. 13. ․ํ 2. Pp. 364-389.

Arroyo, C. (2011). Aproximación al perfil del periodista en la postmodernidad. En [Revista electrónica]. Razón y Palabra. N. ${ }^{\circ}$ 76. Disponible en http://www.razonypalabra.org.mx/N/N76/varia/2a\%20 entrega/25 _ Arroyo _ V76.pdf

Barrios Rubio, A. E Zambrano Ayala, W. R. (2014). Formación de comunicadores "Transmedia" para el público de la generación Digital. Index-Comunicación. Vol. 1. N. 4. Pp. 13-36. Disponible en http://journals.sfu.ca/indexcomunicacion/index.php/indexcomunicacion/article/view/109

Campos, F. (2010). El cambio mediático. Comunicación Social. Sevilla: Zamora.

Castellón, L. y Jaramillo, O. (2009). Competencias digitales para periodistas. En Revista latinoamericana de ciencia de la comunicación (ALAIC). Año VI. N. 10. Pp. 38-51. Disponible en www.alaic. net/revistaalaic/index.php/alaic/article/.../79

Cebrián, M. (2009). Comunicación interactiva en los cibermedios. En Revista Comunicar. Vol. XVII. N. 33. Pp. 15-24.

Cortés, C. (2003). La tecnología de los medios en tiempo de guerra. En Revista Latinoamericana de Comunicación Chasqui. N. ${ }^{\circ}$ 82. Pp. 66-71 
Deuze, M. (2005). What is Journalism? Professional Identity and Ideology of Journalists Reconsidered. Journalism Theory Practice \& Criticism. Vol. 6. N. ${ }^{\circ}$ 4. Pp. 443-465.

Domingo, D. et al. (2007). Four Dimensions of Journalistic Convergence: A preli- minary approach to current media trends at Spain. 8th International Symposium of Online Journalism. University of Texas: Austin.

Franco, G. (2009). El impacto de las tecnologías digitales en el periodismo y la democracia en América Latina y el Caribe. Knight Center for Journalism in the Americas. Disponible en http://knightcenter. utexas.edu/es/node/1012

García, J. (2009). La comunicación ante la convergencia digital: algunas fortalezas y debilidades. En Signo y Pensamiento. Vol. XXVIII. (N. ${ }^{\circ}$ 54). Pp. 102-113.

García, A. y Martínez, O. (2009). Competencias en la formación universitaria de periodistas a través de nuevas tecnologías. En Revista de la Facultad de Ciencias Sociales y Jurídicas de Elche. Vol. I. N. 4 . Pp. 239-250.

González, M. y Ortells, B. (2012). La polivalencia periodística de los profesionales en las redes sociales. En Estudios sobre el mensaje periodístico. Vol. 18. N. ${ }^{\circ}$ Especial. Pp. 455-463.

Hair, J., Bush, R. E Ortinau, D. (2011). Investigación de Mercados 3. a edición. México: McGraw Hill Interamericana, Editores.

Jenkins, H. (2008). Convergence culture. La cultura de la convergencia de los medios de comunicación. Buenos Aires: Paidós.

Larrañaga, J. (2008). La práctica profesional en el proceso de convergencia mediática. Una aproximación metodológica. Estudio de caso del grupo local de comunicación GOIENA Komunikazio Zerbitzuak, Koop.E. En Anàlisi: Quadernsde comunicación i cultura. N. 37. Pp. 93-108.

López, L. (2012). La formación de los periodistas para los entornos digitales actuales. En. Revista de comunicación de la Universidad de Piura. N.¹1. Pp. 278-195.

López, X. E Pereira, X. (2010), Convergencia digital. Reconfiguración de los Medios de Comunicación en España. España: Universidad Santiago de Compostela.

Mcdaniel, C. E Gates, R. (2009). Investigación de Mercados Contemporáneo. 6ta edición. Bogotá: Internacional.

Morales, M. (2005). Tendencias y debates actuales en el periodismo digital. Volver a pensar los medios. En Revista Signo y Pensamiento. Vol. 24. N. ${ }^{\circ}$ 47. Pp. 87-104.

Minsky, M. (2010). The Society of Mind. New York: Simon $\&$ Shuster.

Pavlik, J. V. (2004) A Sea-Change in Journalism: Convergence, Journalists, their Audiences and Sources. En Convergence. N. ${ }^{\circ}$ 10. Pp. 21 - 29.

Quim, S. (2012). Conversations on Convergence. New York: Peter Lang Publishing.

Rey, G. (2013). Medios digitales en Colombia. Estudios de Medios Digitales (EMD). Centro Ático. Pontificia Universidad Javeriana. Inédito.

Rost, A. (2006): La interactividad en el periódico digital. Tesis doctoral. Departament de Periodisme i de Ciències de la Comunicació. Universitat Autònoma de Barcelona.

Salaverría, R. (2005) Convergencia de los medios. En Revista Latinoamericana de Comunicación. Chasqui. N. ${ }^{\circ}$ 81. Pp. 32-39. 
Salaverría, R. (2010) "¿Ciberperiodismo sin periodistas? Diez ideas para la regeneración de los profesionales de los medios digitales". En: Campos Freire, F. (coord.) El cambio mediático. Zamora: Comunicación Social Ediciones y Publicaciones. Pp. 236-249.

Salaverria, R. y Negredo, S. (2008). Periodismo integrado. Convergencia de medios y reorganización de redacciones. Barcelona: Editorial Sol 90.

Salaverria, R. y García, A. (2008). La convergencia tecnológica en los medios de comunicación: retos para el periodismo. Trípodos. N. ${ }^{\circ}$ 23. Disponible en http://www.raco.cat/index.php/tripodos/ article/viewFile/118910/154114

Salazar, M. y Sepúlveda, R. (2011). Perspectivas y proyección profesional de la comunicación social. En Signo y Pensamiento. Vol. XXXI. N. ${ }^{\circ}$ 59. Pp. 194-209.

Scolari, C. (2004). Hacer clic. Hacia una sociosemiótica de las interacciones digitales. Barcelona: Gedisa.

Scolari, C., Micó, J., Navarro, H. y Pardo, H. (2008). El periodista polivalente. Transformaciones en el perfil del periodista a partir de la digitalización de los medios audiovisuales catalanes. Zer: Revista de estudios de comunicación Vol. 13. N.²5. Pp. 37-60.

Túñez, M., Martínez, Y., E Abejón, P. (2010). Nuevos entornos, nuevas demandas, nuevos periodistas. Estudios sobre el mensaje periodístico. Vol. 16 Pp. 79-94. 